\title{
Realization of Solid Plane Cutting System and Reconstruction of Medical 3D Image Based on MC Algorithm
}

\author{
Fei Liu ${ }^{1, a}$ and Dashun Que ${ }^{1,2, b}$ \\ ${ }^{1}$ School of Information Engineering, Wuhan University of Technology, Wuhan 430070, China. \\ ${ }^{2}$ Key Laboratory of Fiber Optic Sensing Technology and Information Processing, Ministry of Education, \\ Wuhan University of Technology, Wuhan 430070, China \\ a2416164702@qq.com, bdsque@sina.com
}

Keywords: MC algorithm, surface rendering, 3D reconstruction, plane cutting.

\begin{abstract}
The three-dimensional reconstruction of medical image is based on the two-dimensional image collection on the imaging device. The reconstructed algorithm can be used to reconstruct and synthesize the three-dimensional images of the lesion or human organ. With the continuous development of medical image three-dimensional reconstruction technology, doctors can be based on the actual condition of the patient's specific diagnosis needs, focus on three-dimensional display of possible lesion area details, can greatly improve the diagnosis rate. Based on the MC algorithm, this paper realizes the reconstruction and 3D visualization system of medical 3D image based on surface drawing using VTK toolkit design. And complete the reconstruction of the zoom, rotation and other interactive operations.
\end{abstract}

\section{Introduction}

Medical image three-dimensional reconstruction is based on the imaging device on the two-dimensional image collection, using computer synthesis to reconstruct the three-dimensional images about the formation of lesion and human organs [1]. So that the human body's internal spatial structure and physical properties can be displayed and widely used in clinical practice. Medical research system is expensive, three-dimensional reconstruction algorithm research cost is high, and the application of medical requirements is very high, and the domestic start in this field late in china, and China's three-dimensional reconstruction algorithm research mainly emphasis on theoretical research. However, compared with the three-dimensional reconstruction software abroad, there are still a single system function, the lack of automatic diagnosis of medical images and other issues.

The surface rendering technique is to set a specific threshold according to the attributes of the target object to be extracted by the user. After extracting the corresponding data points from the body data, we could divided into several triangular patches according to the triangulation. Next using the illumination model in graphics to render the triangular patches to form a three-dimensional image. The key technology is to set the threshold extraction contours as needed. Medical imaging instruments generally output a series of continuous DICOM standard medical image two-dimensional slices, and each slice in the direction $\mathrm{x}$ and direction $\mathrm{y}$ have their own resolution. The Marching Cubes algorithm does not use the algorithm to restore the continuous function, but rather uses the implicit extraction of isometric surfaces. This algorithm does not need to construct the surface function directly, but directly analyzes the volume data, calculates from each voxel information on the face. According to the provided threshold. To extract the soft tissue structure, the corresponding threshold will be reduced. The value distribution may be different. Therefore, the specific CT value of each organization need to be combined with the results of practice and access to the relevant medical literature [2].

\section{MC Algorithm Application and System Frame Design}

For the extracted isosurface, it is necessary to use the graphics algorithm to draw it, and finally get the three-dimensional image. The flow chart of the rebuild system is shown in Fig.1. 


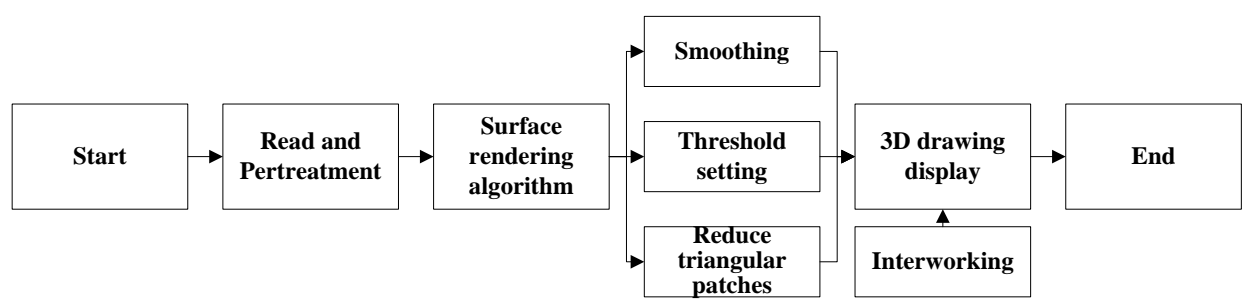

Fig. 1 Three-dimensional reconstruction system

Which involves the graphics rendering and lighting model calculation, the key is to determine the isosurface normal vector. Equivalent surface normal vector determination and point coordinate calculation using linear interpolation and midpoint selection method [3]. (a)Linear interpolation. The formula for linear interpolation is as formula (1). (b)Midpoint selection method. The midpoint selection formula is as formula (2).

$$
\begin{aligned}
& \left\{\begin{array}{c}
P=P_{1}+\left(\text { isovalue }-V_{1}\right) \cdot\left(P_{2}-P_{1}\right) \div\left(V_{2}-V_{1}\right) \\
N=N_{1}+\left(\text { isovalue }-V_{1}\right) \cdot\left(N_{2}-N_{1}\right) \div\left(V_{2}-V_{1}\right)
\end{array} .\right. \\
& \left\{\begin{array}{c}
P=\left(P_{2}-P_{1}\right) \div 2 \\
N=\left(N_{2}-N_{1}\right) \div 2
\end{array} .\right.
\end{aligned}
$$

In the formula(1), P represents the coordinates of equivalent point; P1 and P2 represents the coordinates of the two endpoints; isovalue represents threshold; V1 and V2 represents the gray value of the two endpoints ; $\mathrm{N}$ represents the equivalent point normal vector; $\mathrm{N} 1$ and $\mathrm{N} 2$ represents the normal vector of the two endpoints.

This method directly uses the mean of the two end points in the normal vector or the coordinates as the normal and coordinate values of the threshold. The isosurface is essentially a quadratic surface, so the gradient component of the above point along the tangent direction of the surface is zero, we used the triangular patch to fit it[4]. Thus, the normal vector of the isosurface at that point can be replaced by the direction of the gradient vector of that point. The gradient value on the isoparametric surface is $\mathrm{g}(\mathrm{x}, \mathrm{y}, \mathrm{z})=\nabla \mathrm{f}(\mathrm{x}, \mathrm{y}, \mathrm{z})$, we need to use the Gouraud coloring model, And then draw the triangular patches, so that you can eliminate the black and white between the brightness of the discontinuous changes in the situation [5].The central difference of the vertices are calculated as formula (3).

$$
\left\{\begin{array}{l}
g_{x}=\frac{f\left(x_{i+1}, y_{j}, z_{k}\right)-f\left(x_{i-1}, y_{j}, z_{k}\right)}{2 \Delta x} \\
g_{y}=\frac{f\left(x_{i}, y_{j+1}, z_{k}\right)-f\left(x_{i}, y_{j-1}, z_{k}\right)}{2 \Delta y} . \\
g_{z}=\frac{f\left(x_{i}, y_{j}, z_{k+1}\right)-f\left(x_{i}, y_{j}, z_{k-1}\right)}{2 \Delta z}
\end{array}\right.
$$

\section{System Design Flow and Simulation Implementation}

\subsection{Simulation of Marching Cube Algorithm}

In this paper, a three-dimensional reconstruction system of medical image is completed and an interactive operation is carried out. The experimental data used in the three-dimensional reconstruction of this experiment is 22 images of brain CT with resolution of $512 * 512$. The experimental results are shown in Fig.2.

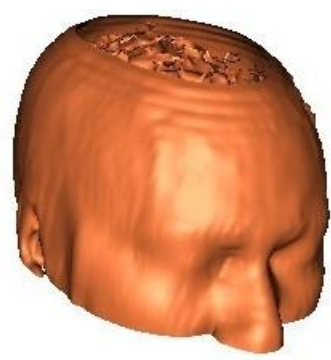

(a) $\mathrm{CT}=200$

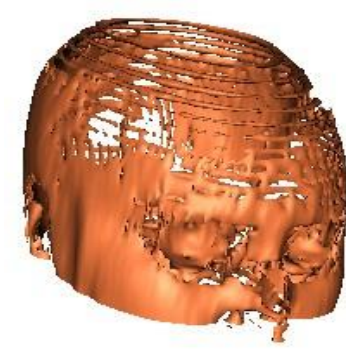

(b) $\mathrm{CT}=600$

Fig. 2 Reconstruction of the brain

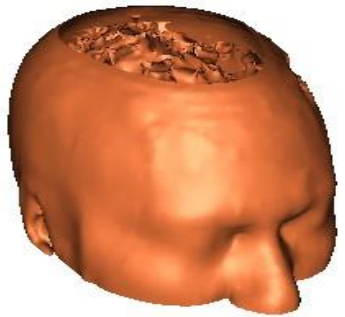

(a )diminished $30 \%$

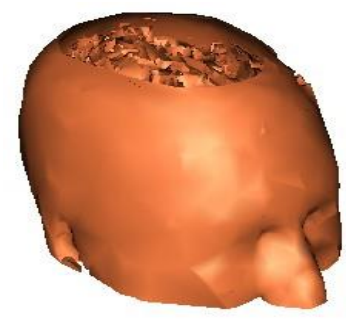

(b)diminished $70 \%$

Fig. 3 Improved for brain reconstruction speed 
From the experimental results of Fig.2 (a) and (b), Since CT images are represented in different gray scale, skin and bone tissue can be extracted according to different gray scale. In the experiment using Setvalue ( $\left.{ }^{*}\right)$ to set the tissue contour gray value. When the gray value of 200 , we can see the skin tissue is extracted; when the gray value of 600 , the head of the skeletal tissue is extracted.

The MC algorithm has achieved three-dimensional reconstruction, but because the algorithm contains a large number of triangular patches, which is not conducive to real-time rendering [6].The flat area is represented by fewer triangles, while in the larger curvature, more triangles are used to increase the triangles of the region to ensure the quality of reconstruction. Using the vtkDecimate base class to achieve triangular slice reduction. The experimental results are shown in Fig.3 (a), (b).

From Fig.3(a) and (b), we can see that when the triangular face is reduced by $30 \%$, the visual effect can be guaranteed. When reduce by $70 \%$, the skin surface with edges and corners, no longer smooth. So we are in the reduction of the visual effect to ensure that the premise, to let the render speed faster. There are also ambiguity problems in the marching cube algorithm that may lead to voids in the resulting equivalent surface. A tetrahedral solution is proposed, but in the case of ambiguity, the resulting triangular patches increase, which is twice the usual cube algorithm [7].

\subsection{Simulation Realization of 3D Solid Surface Cutting}

In this paper, we also set a simulation realization of 3D Solid Surface Cutting, The key to 3D plane cutting is the determination of the cutting plane as its key point. In the three-dimensional coordinate system, the coordinate value $(\mathrm{x}, \mathrm{y}, \mathrm{z})$ can uniquely determine a point in the three-dimensional space, so by setting the slice starting point $(\mathrm{x}, \mathrm{y}, \mathrm{z})$, and then determine the plane normal vector, there is a correspond to determine the central axis, the cutting plane will determine. The geometric transformation matrix of the cutting plane can be expressed as $\mathrm{T}_{3 \mathrm{D}}$.

$$
T_{3 D}=\left[\begin{array}{llll}
a_{11} & a_{12} & a_{13} & a_{14} \\
a_{21} & a_{22} & a_{23} & a_{24} \\
a_{31} & a_{32} & a_{33} & a_{34} \\
a_{41} & a_{42} & a_{43} & a_{44}
\end{array}\right] .
$$

The first nine parameters define the cutting direction of the cutting plane, and the first three parameters of the fourth column determine the entry point. In the VTK provided Widget base class, this experiment uses vtkImagePlaneWidget to create a cutting plane object, successfully demonstrated the coronal and sagittal cutting display. The VTK-based face cutting display is shown in Fig.4.

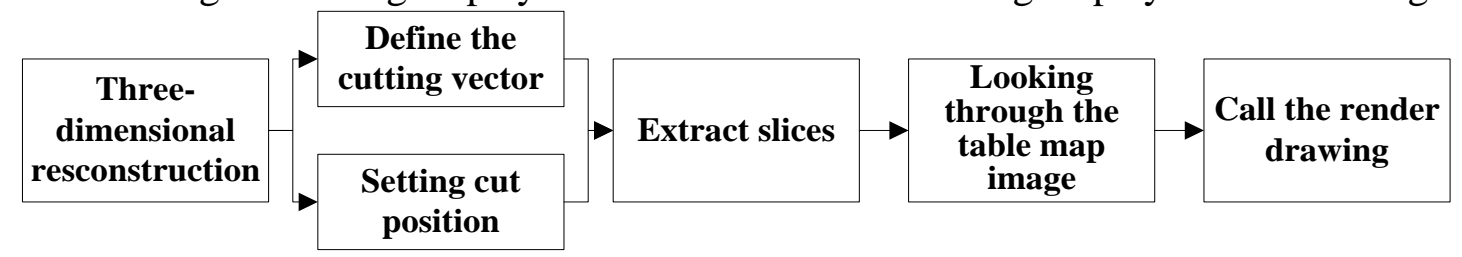

Fig.4 face cut display process

A set of brain tomography sequences were used as experimental data. Reconstructed to achieve sagittal, coronal, cross-sectional display. The experimental results are shown in Fig.5.

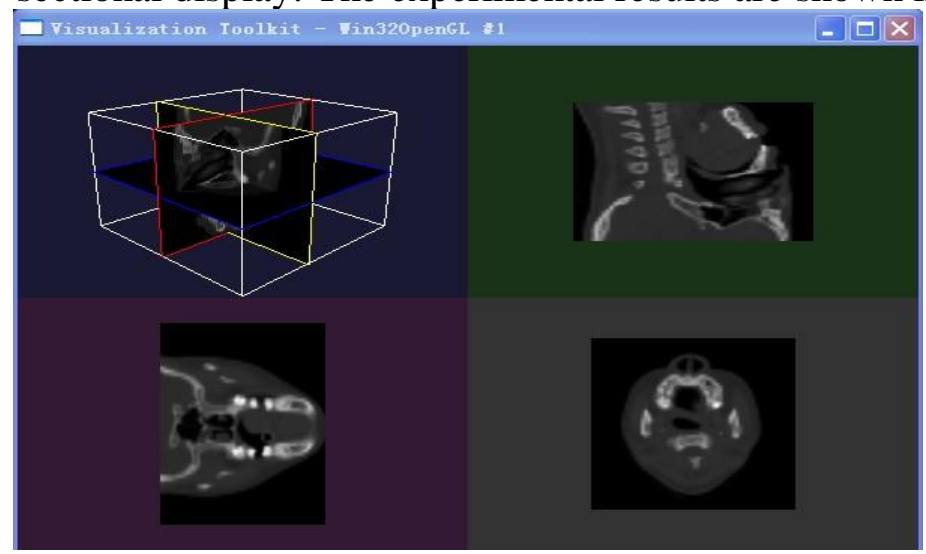

Fig.5 Three-dimensional cutting of the coronal plane, sagittal, cross-sectional display 
From the cutting surface of Fig.5, you can see that the upper left graph is the integrated cross display of each cutting plane; the lower left is the sagittal plane cutting; the upper right is the crown plane cutting effect graph; the lower right is the cross section cutting effect. To achieve from different perspective to study the reconstruction of the body.

\subsection{Other Interactive Functions of the System}

The system is based on the realization of the basic surface rendering algorithm, Fig.6 shows the scaling function of the reconstructions.

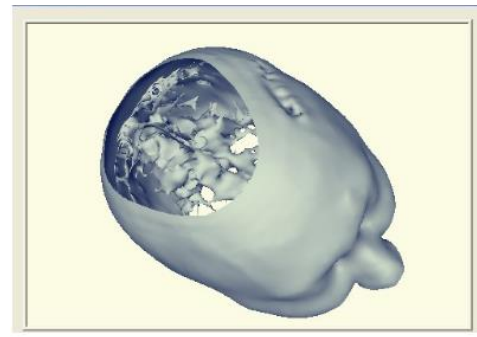

(a) Zoom the head

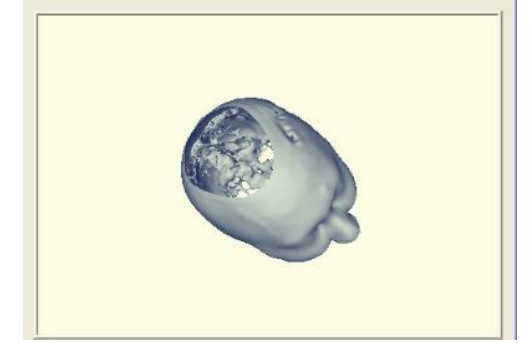

(b) the reduction of the head

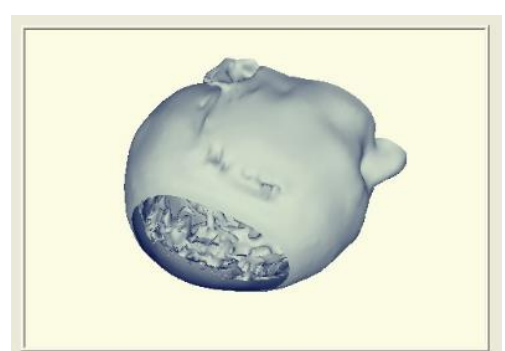

(c) Rotation of the head

Fig. 6 Zoom and rotate functions of 3D reconstructions

The experimental results of Fig.6 (a) and (b) show the amplification and reduction of the reconstructed body. You can click the right mouse button to achieve the three-dimensional reconstruction of the zoom. You can also scroll through the mouse wheel to quickly and easily achieve the zoom function. (C) is by clicking the left mouse button to achieve the rotation of the three-dimensional reconstruction, to overlooking, looking up, side view different effects.

\section{Summary}

Three-dimensional Reconstruction of Medical Images with Existing Surface Rendering and Volume Rendering. Although it realised the three-dimensional reconstruction of the image, but in the drawing speed, drawing effect has yet to be improved. The surface rendering algorithm is better in terms of real-time performance, but it is not ideal for tissue vessels and so on; the volume rendering is just the opposite.so it is necessary to study the mixed drawing algorithm. Although some of the operation and analysis are realized in this paper, the accurate cutting of reconstructed medical images and the accurate measurement of the lesion are needed to be further studied. This has great practical significance on accurate diagnosis, virtual surgery and other clinical medicine.

\section{References}

[1]. Hu Jun-hua, Tian Jin-hui. New Advances in 3D Reconstruction of Medical Image. China Science and Technology Information, Vol. 11 (2013) No. 1, p. 117-117.

[2]. Han Cheng-hu, Han Cheng-long, Wang Meng. Application of MC Algorithm in 3D Reconstruction of CT Medical Image. Modern Computer, Vol 13 (2013) No. 1, p. 20-22.

[3]. Liu Yunwu. Three-dimensional Reconstruction of Medical Image Based on Image Segmentation. Science and Technology Innovation and Productivity,Vol. 34 (2011) No. 1, p. 108-111.

[4]. Li Xiuli. Improvement of cutting algorithm and its application in three-dimensional medical image segmentation. Chinese Academy of Sciences, 2014, p. 10-33.

[5]. Liu Min. Three-dimensional reconstruction of medical image based on VTK. Yanshan University, 2013, p. 18-35.

[6]. HE Yuan-jun, ZENG Wen-quan, YU Ai-min. Review of GPU-based 3D Medical Image Volume Rendering Technology. Journal of Computer and Digital Engineering, Vol. 44 (2016) No. 1, p. 141-147.

[7]. Yang Liang. Several characteristics of grid surface measurement and its application. Zhejiang University, 2014, p. 45-70. 